SINAI Journal of Applied Sciences 9 (1) $2020 \quad 077-088$

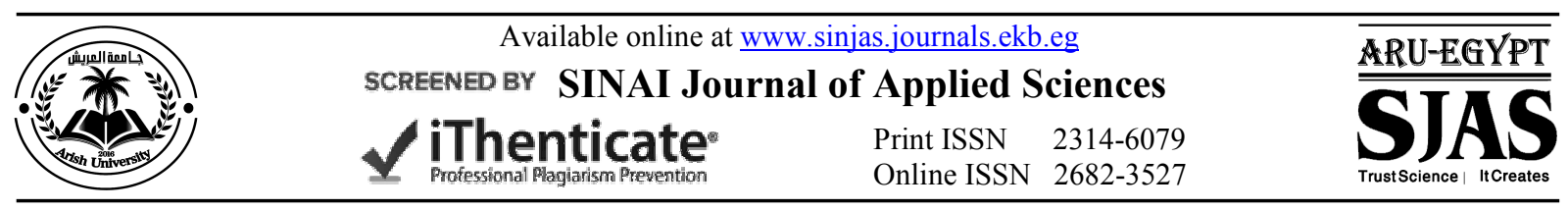

\title{
AN ECONOMIC STUDY OF THE IRANIAN-GULF FOREIGN TRADE AND ITS IMPACT ON THE SAUDI ECONOMY
}

\author{
Hemat M.E. Mahdy ${ }^{1}$ and T.M. Hassanein ${ }^{2}$ \\ 1.Dept. Econ. and Political Sci., Inst. Asian Studies and Res., Zagazig Univ., Egypt. \\ 2.Dept. Agric. Econ., Fac. Agric., Zagazig Univ., Egypt.
}

\begin{tabular}{l} 
ARTICLE INFO \\
\hline Article history: \\
Received: $05 / 01 / 2020$ \\
Revised: $27 / 02 / 2020$ \\
Accepted: $30 / 04 / 2020$ \\
Available online: $02 / 05 / 2020$ \\
\hline Keywords: \\
Foreign trade economics, \\
Saudi economy, \\
Gulf national security, \\
GCC countries.
\end{tabular}

\begin{tabular}{|c|}
\hline Whe \\
Check for \\
updates
\end{tabular}

\begin{abstract}
Achieving Gulf national security has become a major importance for the countries of the Gulf Cooperation Council, especially after the growing challenges they face in this regard, and with the expectation of the emergence of other new challenges with the decrease in the volume of trade exchange between the countries of the Gulf Cooperation Council and Iran, as the volume of trade exchange between Iran and the Arab countries in particular has been affected With the Arab Gulf states with the end of the Arab Gulf War and the emergence of the idea of isolating Iran from commercial exchange with neighboring countries, especially the Arab Gulf states, even though the value of the surplus in the trade balance in Iran amounted to about $\$ 32$ billion annually, While the surplus in the trade balance in the countries of the Gulf Cooperation Council reached about $\$ 422$ billion annually, the value of Iranian exports amounted to about $\$ 72$ billion, while the value of exports in the Gulf Cooperation Council countries amounted to about $\$ 811.2$ billion annually, and the value of Iranian imports amounted to about $\$ 40$ billion and the value of imports in the Gulf Cooperation Council countries reached about \$279 billion annually in 2016, and compared to the volume of trade in Saudi Arabia, which has many natural resources that contribute to the development of its foreign trade movement and that contribute to building many commercial and economic relations with all the countries of the Gulf Cooperation Council, where the value of the Saudi trade volume reached about 13.8 billion Saudi riyals, while the balance of trade reached about 7.6 billion Saudi riyals in 2016, and the study is concerned with the Iranian-Gulf trade exchange and its impact on the Saudi economy despite the strengthening of trade, economic and political relations with Iran.
\end{abstract}

$$
\text { أهمبة البحبل التجارة بين إيران ودول مجلس التعاون الخليجي وما }
$$$$
\text { تكمن أهمية البحث إلى در اسة تطور التبادل التجاري }
$$$$
\text { بين إير ان ودول مجلس التعاون الخليجي و أثر ذلك على البه الته }
$$

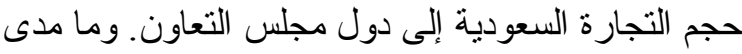$$
\text { أهمية العلاقات الإيرانية السعودية ومعرفة أثز السياسة }
$$$$
\text { الخارجية الإيرانية على التجارة الخارجية في منطقة }
$$$$
\text { الخليج العربي }
$$

\section{المقدمة و المشكية البحثية}

تكمن مشكلة البحث في معرفة مدى حجم التبادل

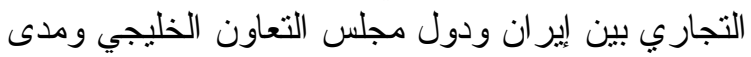

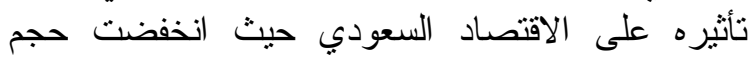

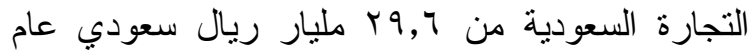

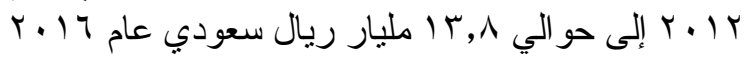

فى ظل ارتفاع حجم التجارة الإير انية مع الدول العربية

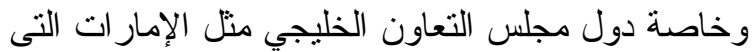

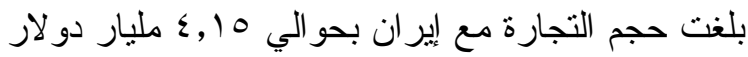

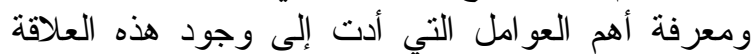

* Corresponding author: E-mail address: https://doi.org/10.21608/SINJAS.2020.86432

(C) 2020 SINAI Journal of Applied Sciences. Published by Fac. Environ. Agric. Sci., Arish Univ. All rights reserved. 
الدول المستوردة من إيران. وبمقارنتها بالكويت تبين ان ان إني

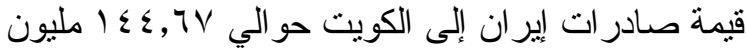

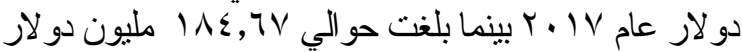

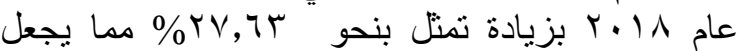

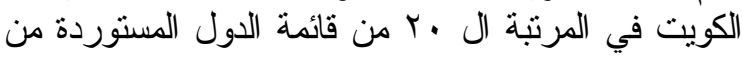

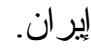

بينما بلغت قيمة صادرات إيران إلى قطر حوالي لئلي

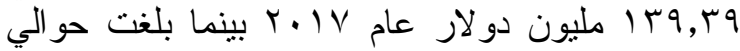

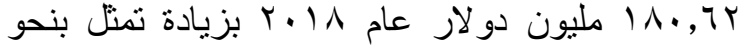

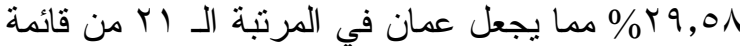

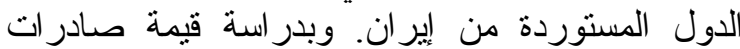

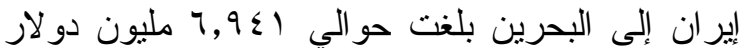

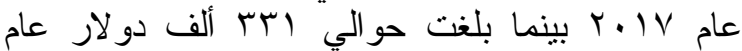

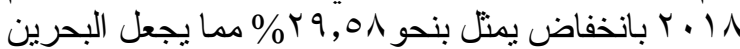

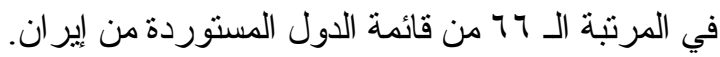

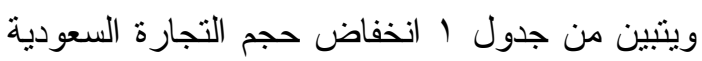

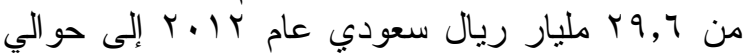

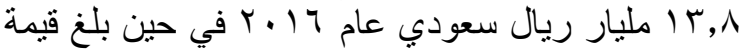

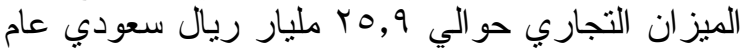

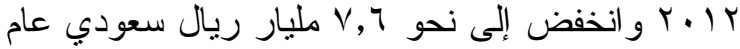

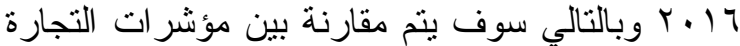
الخارجية لكلا من السعودية و إيران.

\section{در اسة تطور أهم متغيرات التجارة الخارجية بالسعودية}

\section{تطور قيمة الصادرات بالمليار دولار بالسعودية}

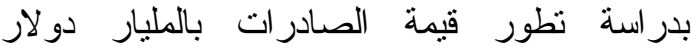

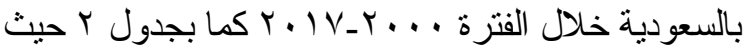

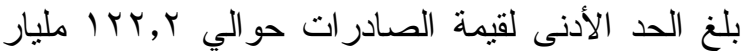

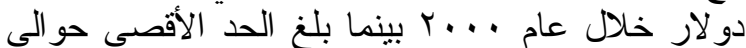

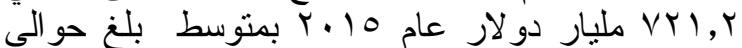

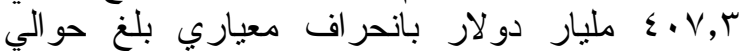

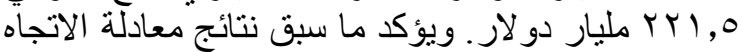

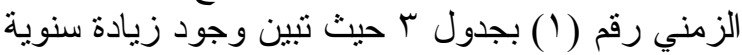

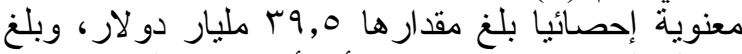

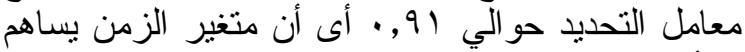

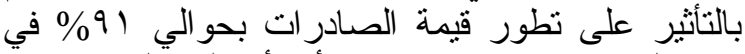

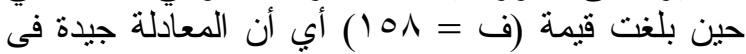

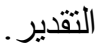

\section{تطور قيمة الواردات بالسعودية}

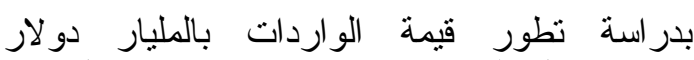

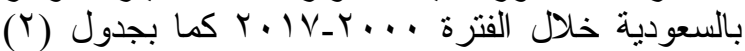

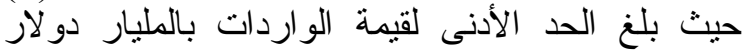

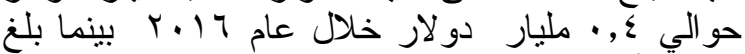

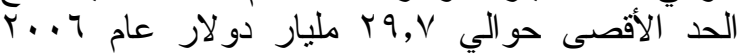

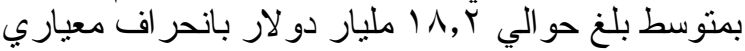

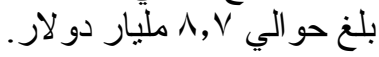

أهد|ف البحث

يهدف البحث إلى النقاط الرئيسية الآتية:

ا ـ در اسة تطور حجم الو اردات السعودية و الإير انية.

r- در اسة تطور حجم الصادر ات السعودية و الإير انية.

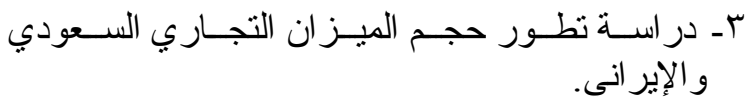

ــ ـمقارنـة بين حجم التجـارة الخارجيـة بين دول مجلس التعاون الخليجي و إير ان.

0ـ در اسة أهم محددات التجارة الخارجية التي تؤنثر على التى الاقتصاد السعودي.

\section{مصادر البيانات والطريقة البحثية}

اعتمد البحث على استخدام المنهج الوصفي و المنهج

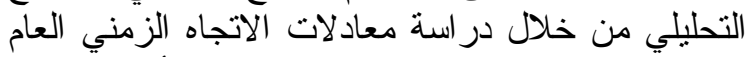
ومعادلات الانحدار البسيط و المتعدد لقياس أثز التر محددات التهات

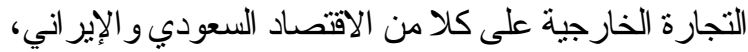

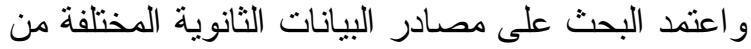

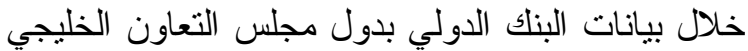

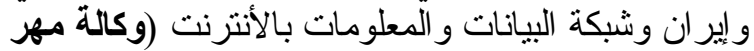

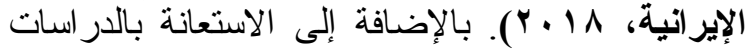
و الرسائل العلمية السابقة بالإضافة إلى الكتب الإنبة والأبحاث العلمية المتعلقة بموضو علئل البحابفة بالاض.

\section{النتائج و المناقشية}

تظور حجم التجارة الإير انية مع دول مجلس التعاون الخليجي

حققت قيمة الصادر ات الإير انية غير النفطبة إلى دول

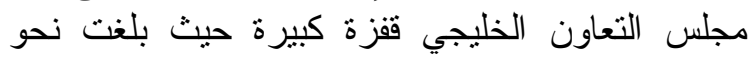

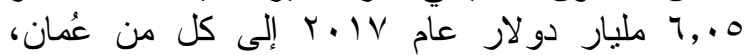

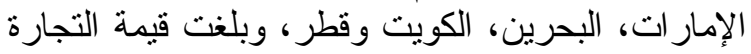

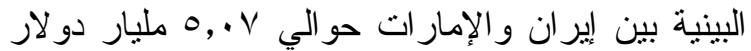

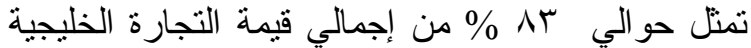

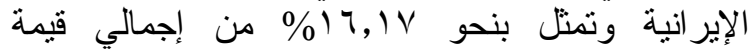

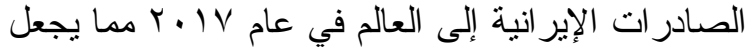

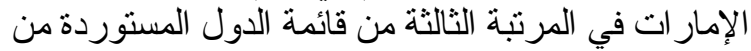

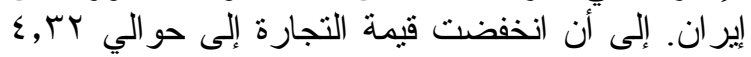

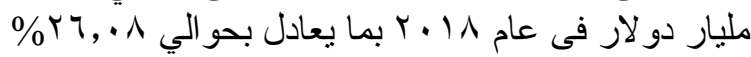

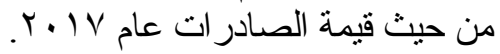

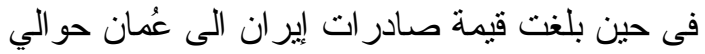

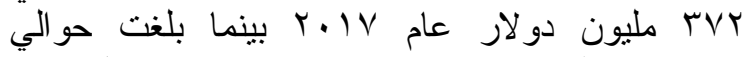

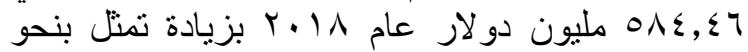
\% ov, II مما يجعل عمان في المرنبة التاسعة من قائمة 
Mahdy and Hassanein | SINAI Journal of Applied Sciences 9 (1) 2020 077-088

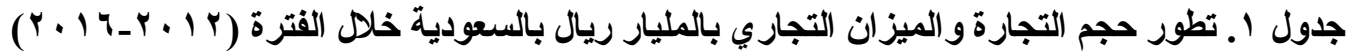

\begin{tabular}{|c|c|c|}
\hline (الميزان التجاري & حجم التجارة & السنوات \\
\hline$r_{0,9}$ & $r 9,00$ & $r+1 r$ \\
\hline$r \varepsilon, r$ & r., ro & $r \cdot I r$ \\
\hline $1 V, 7$ & Yo, Vo & $r .1 \varepsilon$ \\
\hline 0,7 & $1 \leqslant, \vee q$ & $r .10$ \\
\hline$V, \tau$ & $1 \pi, \Lambda$ & $r .17$ \\
\hline 17,11 & $r Y, \wedge Y \wedge$ & المتوسط \\
\hline
\end{tabular}

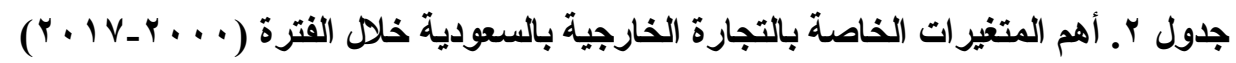

\begin{tabular}{|c|c|c|c|c|}
\hline $\begin{array}{l}\text { (الجمالي الناتج } \\
\text { (مليار دولار) }\end{array}$ & 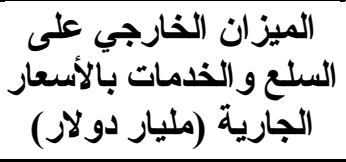 & 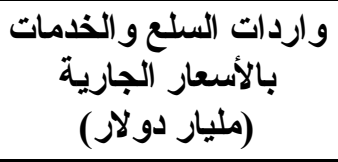 & 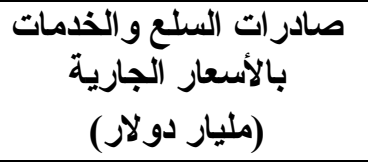 & السنوات \\
\hline$q 1 Y V$ & $1 \cdot r, 7$ & $1 \wedge, 7$ & $M Y Y, Y$ & $r \ldots$ \\
\hline$\Lambda \neg \leqslant Y, 0$ & $11 \cdot, \varepsilon$ & $10, \mathrm{~V}$ & $\mid r 7,1$ & $r \ldots 1$ \\
\hline$\Lambda 700, r$ & $11 \cdot, 9$ & IV,r & IYA, 1 & $r \ldots r$ \\
\hline $907 \mathrm{~V}, 0$ & IYT,Y & Y 1,9 & $1 \leq \wedge, 1$ & $r \ldots r$ \\
\hline$\| r \wedge, \wedge$ & 109 & $r q, q$ & 110,9 & $r \ldots \varepsilon$ \\
\hline I rVYq,A & $r .0, T$ & Yr, & YTV,V & $r \ldots o$ \\
\hline IOYYE,V & roq & $r q, v$ & $r \wedge \wedge, \vee$ & $r \ldots q$ \\
\hline $1 T \leqslant V Y, Y$ & $r \leqslant r, V$ & ro & $r ૫ \Lambda, V$ & $r \ldots v$ \\
\hline$r \ldots r v, \wedge$ & $\varepsilon) 7, \varepsilon$ & rA, $)$ & $\varepsilon \varepsilon \varepsilon, 0$ & $r \ldots \Lambda$ \\
\hline $17.9 \varepsilon, r$ & $\varepsilon \cdot 0, \mu$ & $q, r$ & $\varepsilon 1 \leqslant, 0$ & $p \ldots q$ \\
\hline $19 r 09,7$ & س, & 17,7 & $\varepsilon \wedge r, q$ & $r \cdot 1$. \\
\hline$r Y r V \cdot, \Lambda$ & $0 \leqslant r, r$ & rT, & 071,1 & 5.11 \\
\hline ror.r,l & $0 \wedge 9,9$ & ro & $7) \leq, 9$ & $r .1 r$ \\
\hline$r \leq q r \varepsilon, r$ & $7 \leq 1, \varepsilon$ & YI, & OT,O & $r .1 r$ \\
\hline$r \leq 0 \vee 0, \varepsilon$ & $V \cdot r, r$ & $|r|$, & $V) \leq, r$ & $r \cdot 1 \leq$ \\
\hline$r \cdot V r r, q$ & $v 10,9$ & $0, \xi$ & VYI, & $r .10$ \\
\hline $199 \wedge r, 1$ & r & $\cdot, \Sigma$ & $T r q, V$ & $r .17$ \\
\hline$r \cdot V_{T} \cdot, q$ & OVT, Y & $0, \Sigma$ & $0 \wedge 1,7$ & $r+I r$ \\
\hline $17994, V$ & 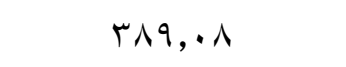 & $1 \wedge, r$ & $\varepsilon \cdot v, r$ & المتوسط \\
\hline $0 \wedge 79,1$ & r rO, r & $\wedge, \vee$ & rrI,0 & الالمعراف \\
\hline$\Lambda T \leqslant r, 0$ & $1 \cdot r, 7$ & $\cdot, \varepsilon$ & Irr, & الحد الأدنى \\
\hline ror. r, I & $v 10,9$ & $r q, V$ & VYI, & الحد الأعلى \\
\hline
\end{tabular}


تؤثر على كمية الصادر ات و الواردات من و الى السعودية من دول المجلس.

\section{در اسة تطور أهم متغير ات التجارة الخارجية بإيران}

تطور قيمة الصادرات بالمليار دولار بايران:

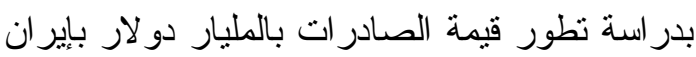

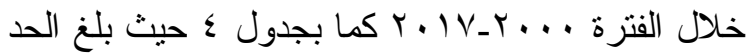

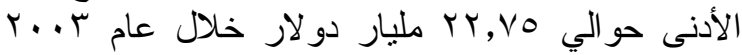

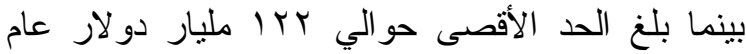

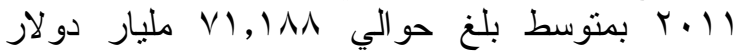

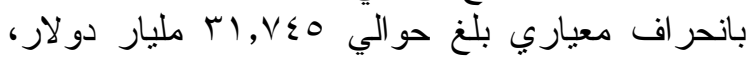

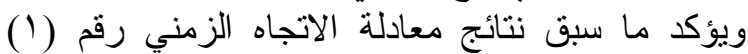

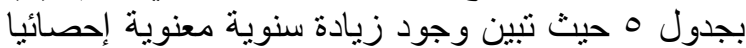

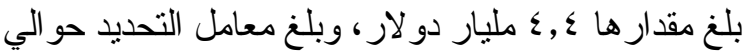

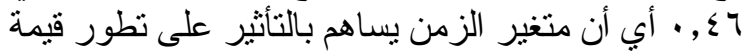

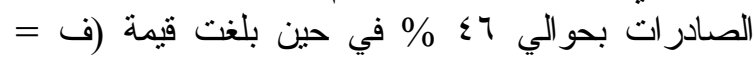
(T),V

\section{تطور قيمة الو اردات باييران}

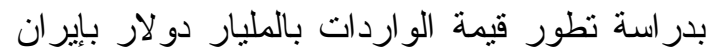

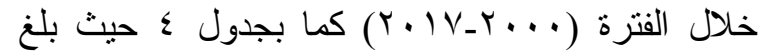

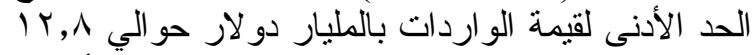

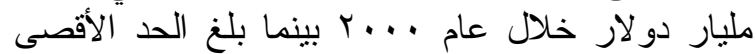

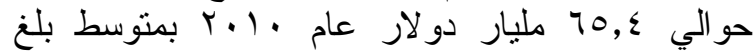

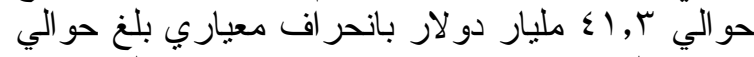

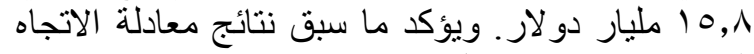

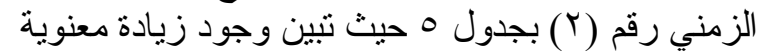

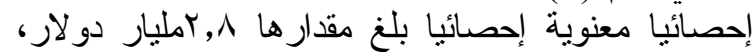

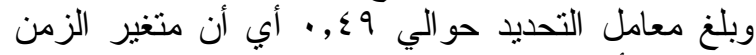

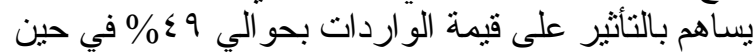

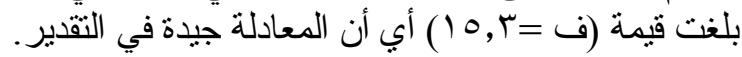

\section{تطور قيمة الميزان التجاري بإيران}

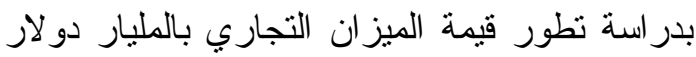

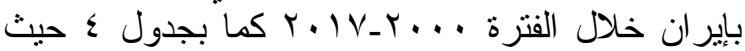

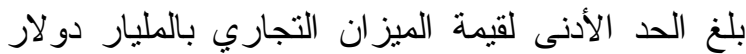

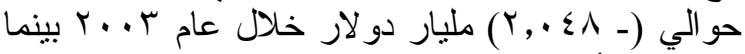

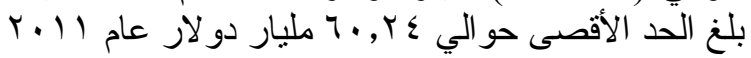

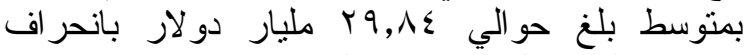

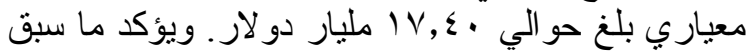

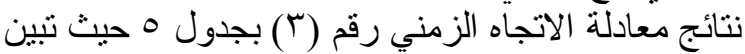

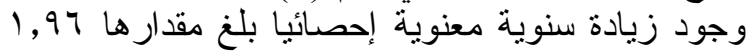

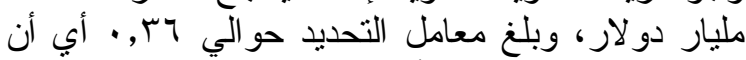

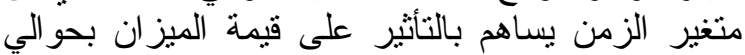

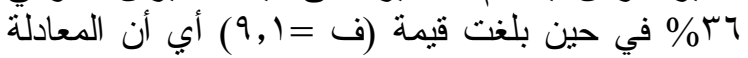
جيدة فى التقدير.
ويؤكد ما سبق نتائج معادلة الاتجاه الزمني رقم (r)

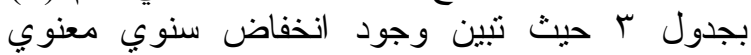

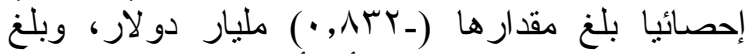

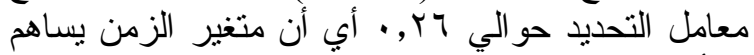

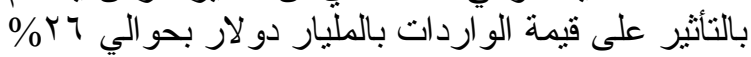

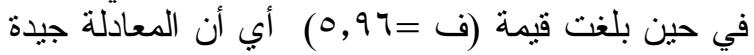
في التقدير.

\section{تطور قيمة الميزان التجاري بالسعودية}

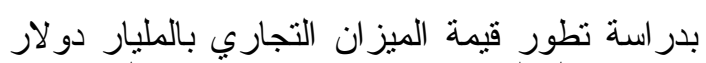

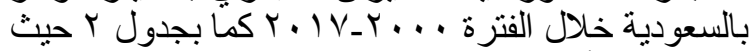

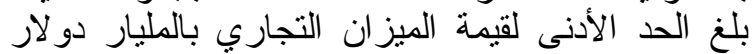

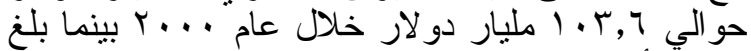

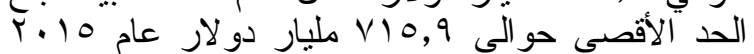

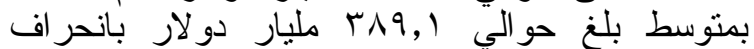

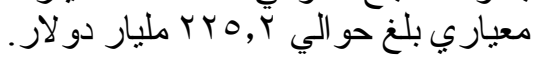

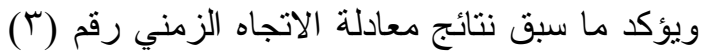

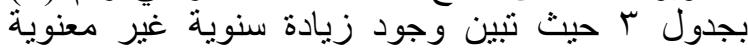

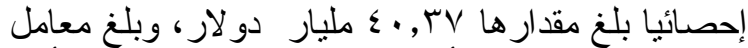

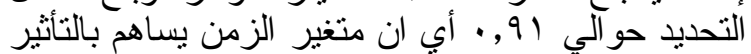

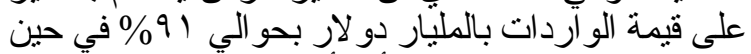

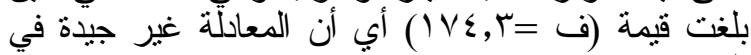
الثقدير.

\section{تطور قيمة الناتج القومي بالمليار دولار بالسعودية}

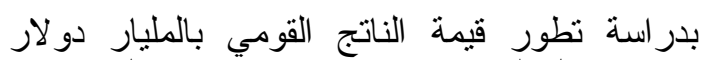

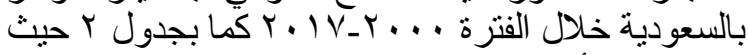

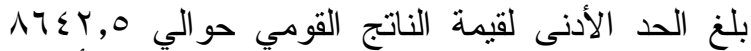

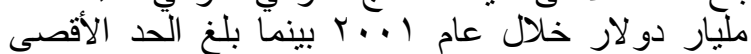

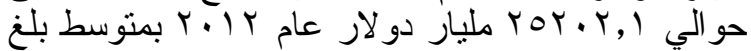

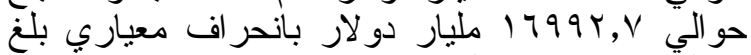

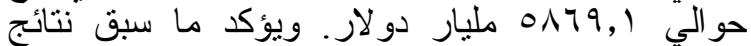

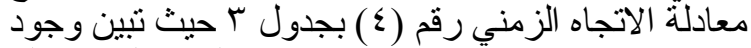

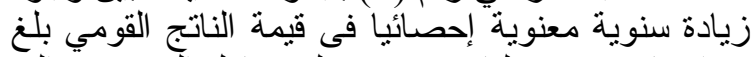

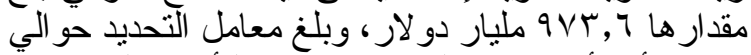

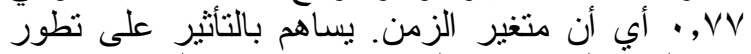

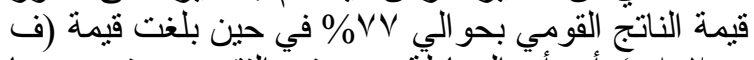

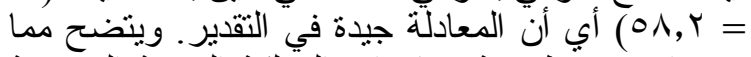

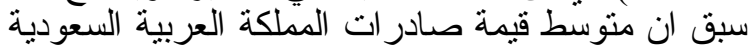

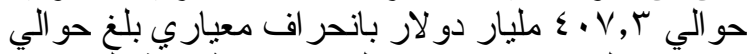

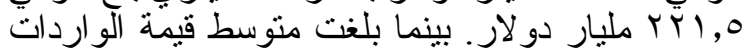

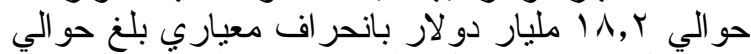

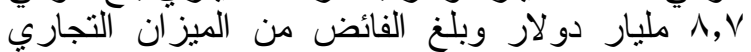

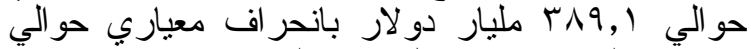

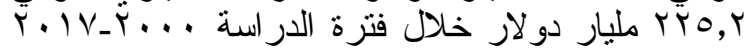

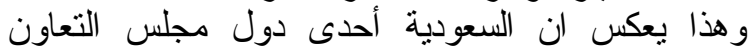

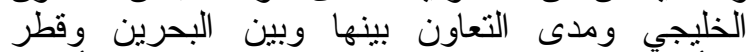
و الأمار"ات و عمان و الكويت وحينما تتخل دول دول أخرى

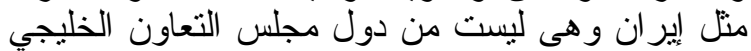
بعلاقتها العسكرية و التجارية مع تلك الدول فإنها سوف التهاري 
Mahdy and Hassanein | SINAI Journal of Applied Sciences 9 (1) 2020 077-088

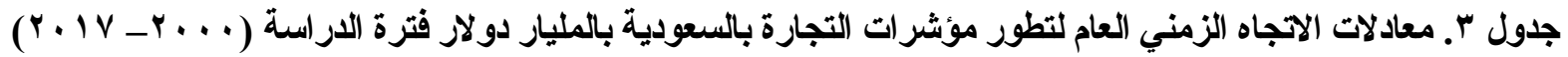

\begin{tabular}{|c|c|c|c|c|c|c|c|}
\hline مغنوية & معنوية & التحديد & معدل التغير & $\mathbf{B}^{\wedge}$ & $\mathbf{a}^{\wedge}$ & البيان & p \\
\hline$*(101)$ & $*(1, Y, 7)$ & $\cdot, 91$ & $9, v$ & $r q, 0$ & 41,09 & قيمة الصادر ات & 1 \\
\hline$*(0,97)$ & $*(\Upsilon, \Gamma \wedge-)$ & Y & $\varepsilon, 7$ & - & Y & قيمة الواردات & $r$ \\
\hline$*(\mid \vee \varepsilon, \Gamma)$ & $*(\mid r, r)$ & $\cdot, 91$ & $1 \cdot, \uparrow \wedge$ & $\varepsilon \cdot, r V$ & $0,0 Y$ & الميزان التجاري & $r$ \\
\hline$*(\diamond \wedge, r)$ & $*(\vee, \uparrow)$ & $\cdot, \vee V$ & $0, v$ & $9 \vee r, 7$ & $V V, \varepsilon r$ & الناتج القومي & $\varepsilon$ \\
\hline
\end{tabular}
y المصدر: حسبت بجدول r باستخدام برنامج التحليل الإحصائي EXCEL.

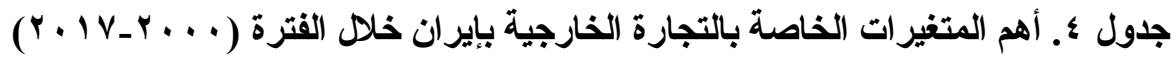

\begin{tabular}{|c|c|c|c|c|c|c|c|c|}
\hline فع الناتج النمو & 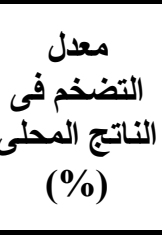 & 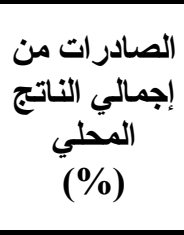 & 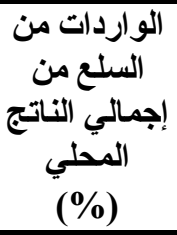 & إلقومي (مليار & 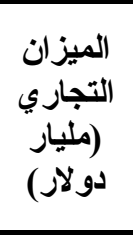 & بالأسعار الجاريّة & بالأسعار الجارية الدية & السنوات \\
\hline 0,9 & $r \varepsilon, 9$ & $r 1,0$ & 19,1 & $1.9,095$ & $10, \wedge{ }_{1}$ & $1 Y, \wedge q \wedge$ & $r \wedge, V Y q$ & $r \ldots$ \\
\hline$\cdot, \wedge$ & 17,7 & $19, \mathrm{r}$ & YI, r & $1 K 4, A \vee q$ & $\wedge, 9 \wedge$ & $17,7.9$ & ro, T/q & $r \ldots l$ \\
\hline$v, r$ & $r q, r$ & $r \leq, \varepsilon$ & $r Y, \Lambda$ & IKA,TrV & $r, \wedge r T$ & $r \cdot, T) V$ & $r \leqslant, \varepsilon \varepsilon$ & $r \ldots r$ \\
\hline$\wedge, \vee$ & Ir & $r \varepsilon, T$ & rז, & $104,0 \leqslant 0$ & $r, \cdot \leqslant \wedge-$ & $r \varepsilon, \vee ৭ \wedge$ & Yr,VO & $r \ldots r$ \\
\hline$\varepsilon, \varepsilon$ & $r \leq, V$ & ro,r & Y & $19 ., ., \leqslant Y$ & $|9, V Y|$ & YI,qV५ & $\leqslant 1,79 \vee$ & $r \ldots \varepsilon$ \\
\hline$r, r$ & $r \cdot, r$ & $r \cdot, r$ & $r \varepsilon, 1$ & KYT, \&OY & $17, r 11$ & $\{\cdot, \cdot \leqslant 1$ & OT, ror & r... \\
\hline 0 & $1 \leqslant, 7$ & $r q, q$ & rY, Y & & Tצ, Y & $\varepsilon,, V \vee r$ & $V V, \cdot 1 Y$ & $r \ldots r$ \\
\hline$\Lambda, r$ & rr,q & $r \Lambda, \Lambda$ & rI & $r \leqslant q, \wedge \wedge r$ & $\varepsilon r, \vee \wedge$ & $\varepsilon \varepsilon, 9 \leqslant Y$ & AN, VYY & $r \ldots v$ \\
\hline$\cdot, r$ & $19, \varepsilon$ & ז & $r,, V$ & $\{.7, \cdot V\}$ & $00, Y \backslash V$ & ov, $\varepsilon+1$ & גו'ו & $r \ldots \wedge$ \\
\hline 1 & צ, & rr,, & rI & $\{1 \leqslant, .09$ & rA,.Or & $0 ., V 71$ & VA,Ar & $r \ldots q$ \\
\hline $0, \wedge$ & 10,9 & $r \leqslant, \varepsilon$ & $19, \varepsilon$ & $\varepsilon \wedge \vee, \cdot \vee$ & $r_{0, \Lambda}$ & $70, \varepsilon \cdot \varepsilon$ & $1 \cdot 1, r 14$ & $r+1$. \\
\hline$r, \uparrow$ & rY, & $r_{0, T}$ & $1 Y, T$ & ONY,O & $T \cdot, Y \leq$ & $ד, \vee \Upsilon$ & IrT & $r+11$ \\
\hline$v, \varepsilon_{-}$ & $r \varepsilon$ & $r \leqslant, 1$ & rr, r & O৭৯,人०Y & $\leq 7,9 \cdot 1$ & OV,.9Y & $1 \cdot \varepsilon$ & $r \cdot T^{\prime}$ \\
\hline$\cdot, Y_{-}$ & $r_{0, T}$ & r & rY, $\varepsilon$ & $\leqslant \tau V, \varepsilon 10$ & rT,r & $\varepsilon १, r$ & Nr,o & $r .1 T$ \\
\hline$\leq, 7$ & $1 \cdot, \wedge$ & $r, Y$ & $r_{1,0}$ & $\varepsilon r \varepsilon, \varepsilon V_{0}$ & $r v, \wedge$ & 01 & $\wedge \wedge, \wedge$ & $r+1 \leq$ \\
\hline $1, Y_{-}$ & •, § & 19,8 & $19, \mathrm{r}$ & rso, Avo & $r \cdot, r$ & $\varepsilon 1, \wedge$ & Tr, & $r+10$ \\
\hline$I^{\prime}, \varepsilon$ & 1,7 & 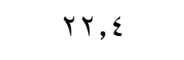 & $r \cdot, \wedge$ & $\varepsilon 1 \Lambda, q \vee \vee$ & rr & $\varepsilon$ & VY & $r .17$ \\
\hline$\varepsilon, r$ & $\wedge, 1$ & $r Y, \Lambda$ & YY, & SOr, . Vq & $\leq 0$ & $\leq V$ & 9र & $r \cdot I V$ \\
\hline$r, 0 \wedge$ & 17,07 & $r r, 0$ & $r,, V r$ & & $r q, \wedge \leqslant 0$ & $\varepsilon 1, r \leqslant r$ & $\mathrm{~V}, 1 \mathrm{MN}$ & المتوسط \\
\hline$\varepsilon, \varepsilon V$ & $\Lambda, V^{\prime}$ & r,97 & $r, I V$ & 101,179 & $\mid v, \varepsilon .0$ & 10,ANr & $r 1, v \leqslant 0$ & المعياري \\
\hline$V, \varepsilon_{-}$ & $\cdot, \varepsilon$ & $19, r$ & IV,T & $1.9,094$ & $r, \cdot \leq \wedge-$ & $\mid r, \wedge 91$ & rY,YO & الحد الأنىى \\
\hline $1 Y, \varepsilon$ & $r q, q$ & $r q, 9$ & $r 4,1$ & 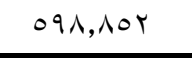 & $T \cdot, Y \varepsilon$ & $70, \varepsilon \cdot \varepsilon$ & IrT & الحد الأعلى \\
\hline
\end{tabular}




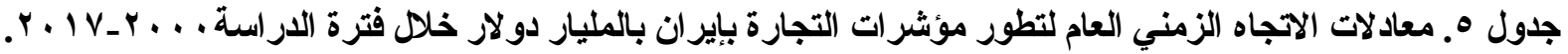

\begin{tabular}{|c|c|c|c|c|c|c|}
\hline معنوية & معنوية & التحديد & $\mathbf{B}^{\wedge}$ & $\mathbf{a}^{\wedge}$ & البيان & s \\
\hline$*(\mid \Gamma, \vee)$ & $*(\Upsilon, \vee)$ & $\cdot, \leqslant 7$ & $\varepsilon, \varepsilon$ & $r r, v q$ & قيمة الصادرات (المليار دولار) & $T$ \\
\hline$*(10, r)$ & $*(\Gamma, 9)$ & $\cdot, \leqslant 9$ & $r, \wedge$ & YI,OV & قيمة الواردات(المليار دولار) & r \\
\hline$*(9,1)$ & $*(\Gamma, \cdot)$, & צו, & 1,97 & $11, r Y$ & الميزان التجاري (المليار دولار) & $r$ \\
\hline$*\left(Y_{\odot, \wedge}\right)$ & $*(0, \vee)$ & ז & דr, & $11 \cdot, 74$ & الناتج القومي (المليار دولار) & $\varepsilon$ \\
\hline$(Y, 19)$ & $(1, \Sigma \wedge)$ & $\cdot, \operatorname{lr}$ & $\cdot, 1 \leq-$ & $r r, \cdot V$ & الو اردات من إجمالي الناتج المحلى(\%) & ○ \\
\hline$(\cdot, \cdot 1)$ & $(\cdot)-)$, & $\cdot, \cdots$ & $\cdot, \cdot 1-$ & $r \leqslant, \cdot 9$ & الصادرات من أجمالي الناتج المحلى (\%) & 9 \\
\hline$*(\neg, \vee)$ & $*(\Upsilon, O \wedge-)$ & $\cdot, r \cdot$ & $\cdot, \wedge \wedge$ & $r \varepsilon, 9 \wedge$ & تطور معدل التضخم في الناتج القومي الإيراني (\%) & $v$ \\
\hline$(\cdot, \leqslant\rceil)$ & $(\cdot, \uparrow \wedge-)$ & $\cdot, \cdot r$ & $\cdot, 1 \leq-$ & $\varepsilon, 9)$ & معدل النمو في الناتج المحلى (المليار دولار) & $\Lambda$ \\
\hline
\end{tabular}

المصدر: حُسبت من بيانات جدول (ع) ) باستخدام برنامج التحليل الإحصائي EXCEL.

صادرات السلع من إجمالي الناتج المحلى:

بدر اسة تطور تطور صادر ات السلع من إجمالي الناتج

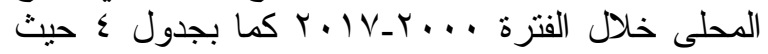

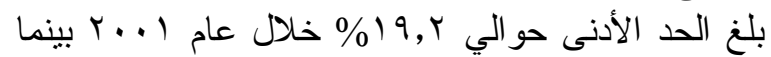

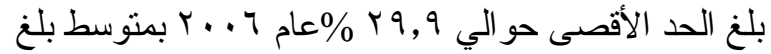

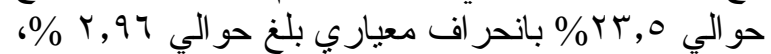

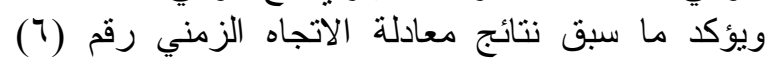

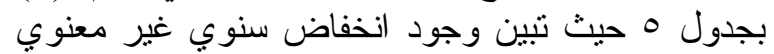

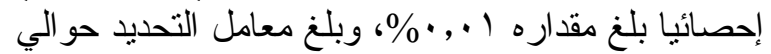

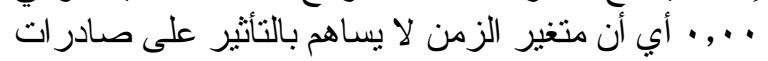

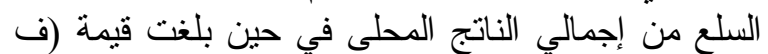

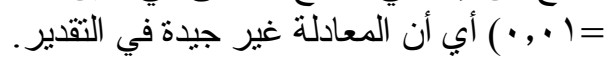

\section{تطور معدل التضخم فى الناتج القومي الإيراني}

بدر اسة تطور معدل التضخم فيى الناتج القومي

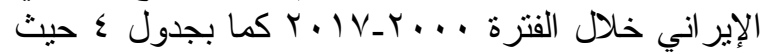

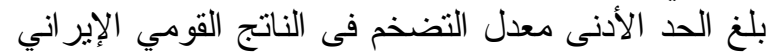

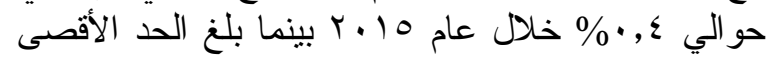

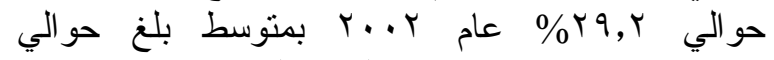

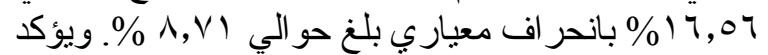

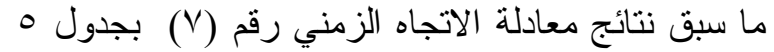
حيث تبين وجود انخفاض سنوي معنوي إحصائيا لتئي في

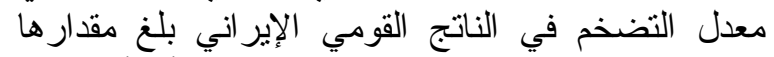

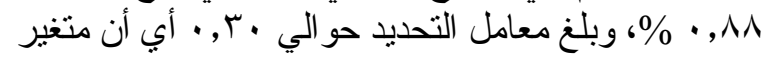

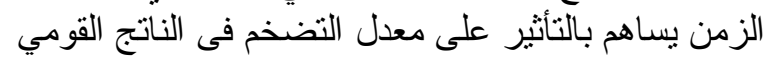

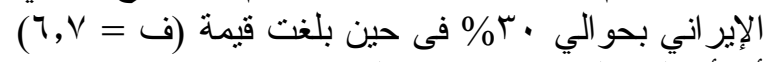
أي أن المعادلة غير جيدة فى التقدير.
تطور قيمة الناتج القومي بالمليار دولار بإيران

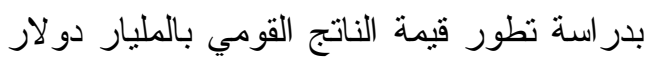

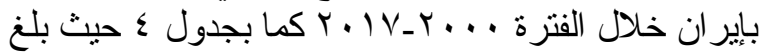

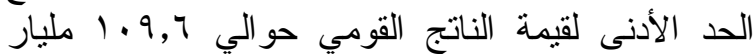

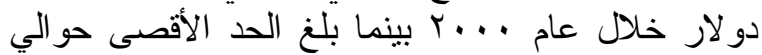

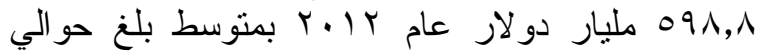

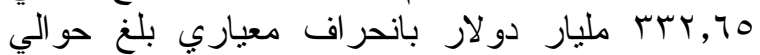

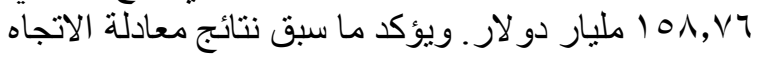

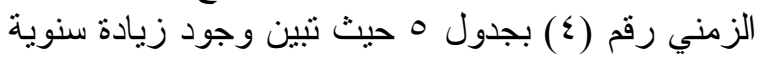

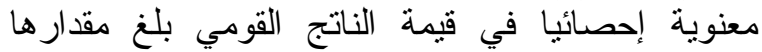

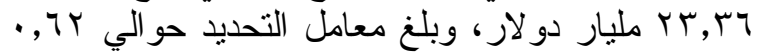

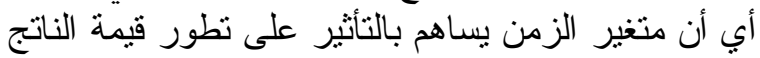

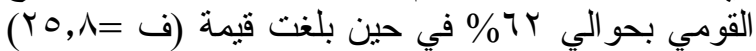
أي أن المعادلة جيدة في التقدير .

\section{تطور واردات السلع من إجمالي الناتج المحلى لئل}

بدر اسة تطور مؤشر واردات السلع من إجمالي الناتج

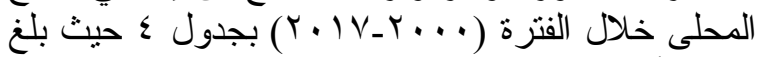

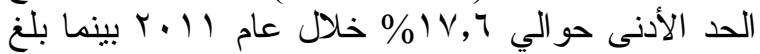

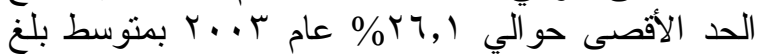

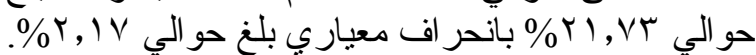

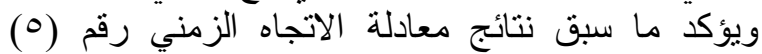

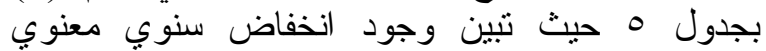

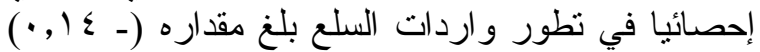

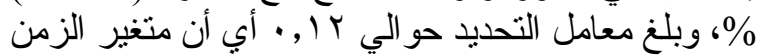

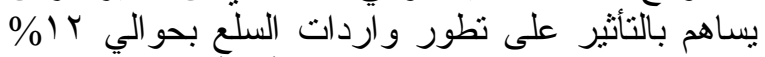

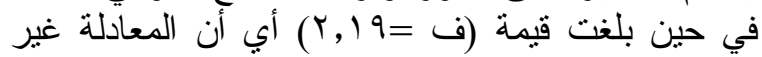
جيدة في التقدير. 
البحرين وقطر و الأمار ات و عمان و الكويت و السعودية

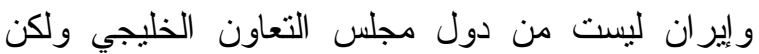

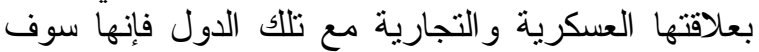

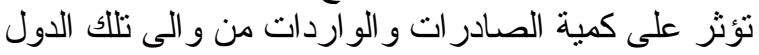
و هذا يعكس مدى تطور نشاط إير ان عسكريأ و اقتصادياً.

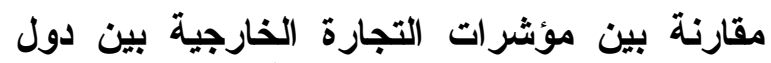
مجلس التعاون الخليجي و السعودية و إيران

يتضح من جدول 7 أهم مؤشرات التجارة الخارجية

بين دول مجلس التعاون الخليجي وكلا من النئن السعودية

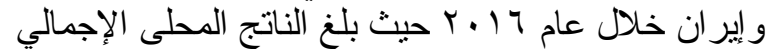

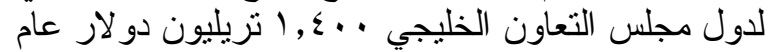

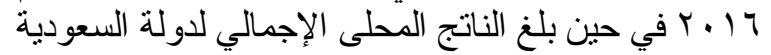

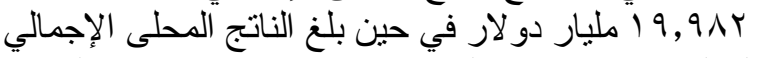

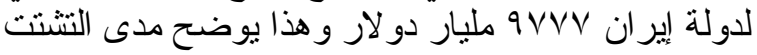

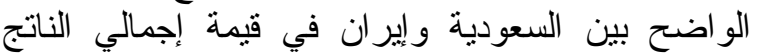

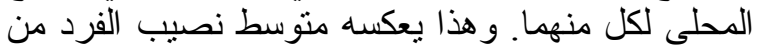

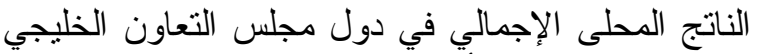

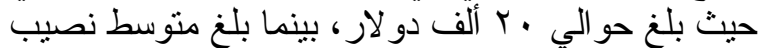

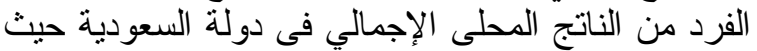
بلغ حوالي گه ألف دولار سنويا. في حين بلغ الإلغ منوسط نصيب الفرد من الناتج المحلى الإجمالي في دولئ دولة إيران حو الي ا, با ألف دولار سنويا
تطور معدل النمو فى الناتج المحلى الإيراني

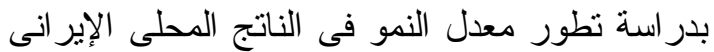

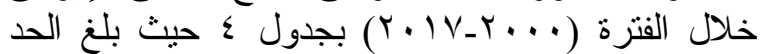

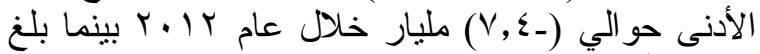

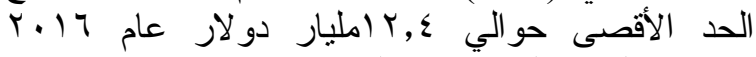

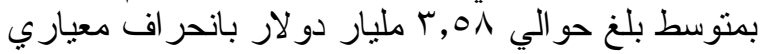

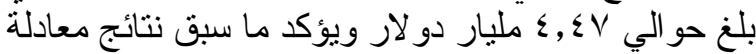

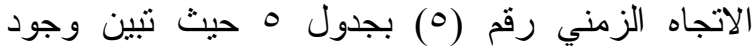

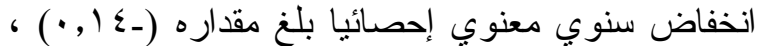

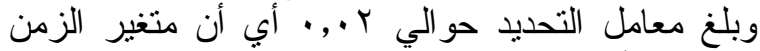

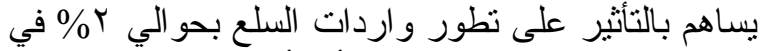
حين بلغت قيمة (ف =7 ؟ ـ • ) أي أن المعادلة غير جيدة

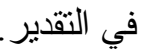

يتضح مما سبق أن متوسط كمية صادر ات إير ان بلغت

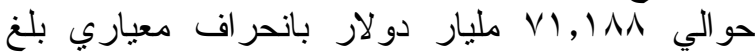

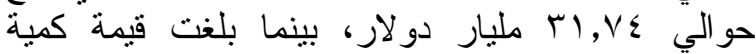

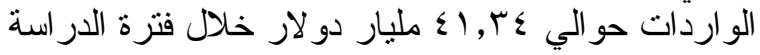

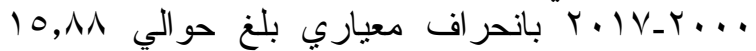

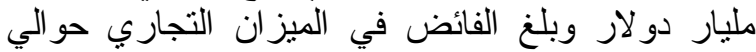

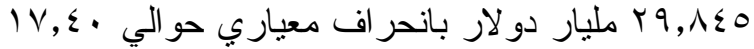

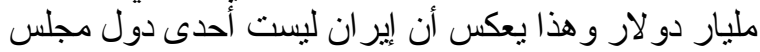
التعاون الخليجي وهذا يعكس مدى الإيل التعاون بينها وبين دلين

جدول T. أهم مؤشر ات التجارة الخارجية بين دول مجلس التعاون الخليجي و إيران خلال عام 17 ـ ץ.

\begin{tabular}{|c|c|c|c|}
\hline إيران & السعودية & دول مجلس التعاون الخليجي & البيان \\
\hline مليار دو لار سنوياً VqVV ملياً & ب91991 مليار دولار سنوياً & 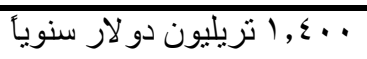 & الناتج المحلى الإجمالي \\
\hline ا, ب, ألف دو لار سنوياً & ـه ألف دو لار سنويا & • ب ألف دو لار سنوياً & نصيب الفرد من إجمالي الناتج المحلى \\
\hline r, ب, مليار برميل يومياً & ك, ب, مليار برميل يومياً & ب r, ا (مليار برميل يومياً & الإتتاج النفطي \\
\hline V r ا مليار برميل & Vll المليار برميل & 1, ד 9 § مليار برميل & الاحتياطي النفطى \\
\hline بـ ا مليار متر مكعب سنوياً & هـ 1 مليار متر مكعب سنوياً & 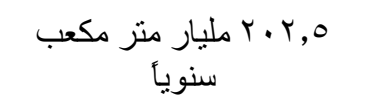 & الإنتاج من الغاز الطبيعي \\
\hline- & - & 1, إ مليار متر مكعب & احتياطي الغاز الطييعي \\
\hline بـ مليار دو لار سنوياً & r, ז זr مليار دو لار سنوياً & r r مليار دو لار سنوياً & الفائض فى الميزان التجاري \\
\hline اليار دو لار & ا & r, 1ו1 مليار دو لار & إجمالي الصادرات \\
\hline • ع مليار دو لار سنوياً & ـ ـ, • مليار دو لار سنوياً & l Y Vq & إجمالي الواردات \\
\hline 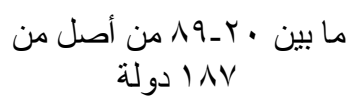 & 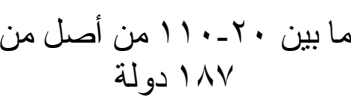 & 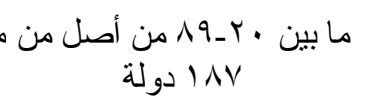 & معدل التتمية البشرية \\
\hline
\end{tabular}

المصدر: الأمانة العامة لمجلس التعاون الخليجي العربي، التقرير الإحصائى السنوي، 17 +؟ب. 
E3

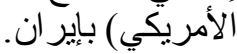

E

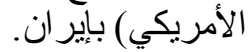

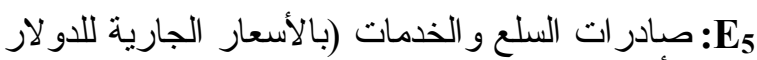

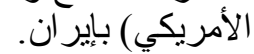

E6 الجارية للاو لار الأمريكي) بإيران.

E7

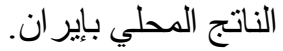

$$
\text { الثكل الرياضى للنموذج }
$$

يأخذ النموذج الصورة التالية:

$\mathrm{Y}_{\mathrm{E}}=\mathrm{a}_{0}+\mathrm{a}_{1} \mathrm{E}_{1}+\mathrm{a}_{2} \mathrm{E}_{2}+\mathrm{a}_{3} \mathrm{E}_{3}+\mathrm{a}_{4} \mathrm{E}_{4+} \mathrm{a}_{5} \mathrm{E}_{5}+\mathrm{a}_{6} \mathrm{E}_{6}+\mathrm{a}_{7} \mathrm{E}_{7}$

تقدير العلاقة الخطية بين أهم المتغيرات التي تؤثر

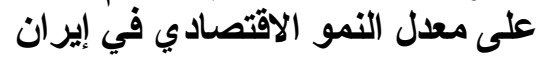

سوف يتم تقدير العلاقة بين أهم المتغير ات التير التي نؤنثر

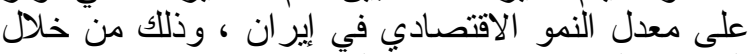

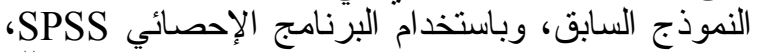

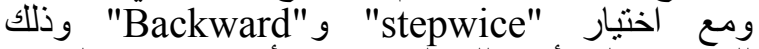
للوقوف على أهم تلك المتغير ات أهم محددات التجارة الخارجية على التي تؤثر على النمو الاقتصادي في إير اند).

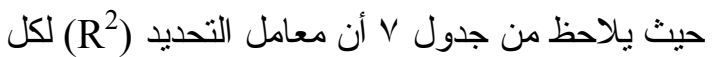

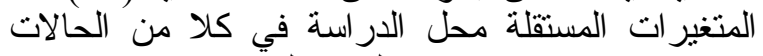

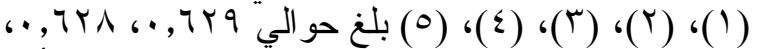

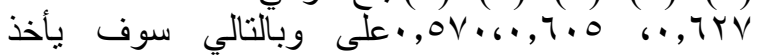

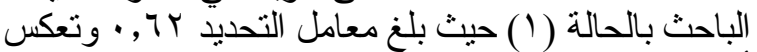

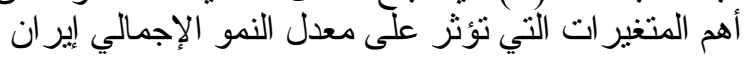

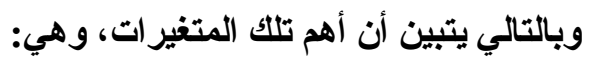
نسبة مساهمة القيمة المضافة من الصناعات التحويلية من معدل النمو بإير ان.

: E2 الإجمالي بإيران.

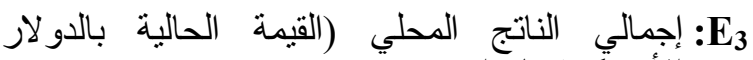
الأمريكي) بإيران.

E:

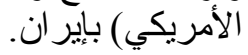

E5: صادر ات السلع و الخدمات (بالأسعار الجارية للدو لار

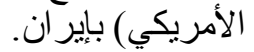

E6 الجارية للاد لار الأمريكي) بإير ان.

E7

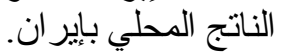

وبلغ الإنتاج النفطى بدول مجلس التعاون الخليجي

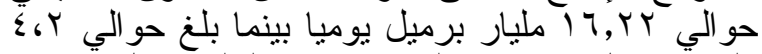

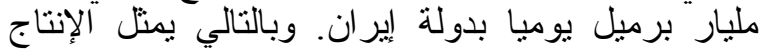

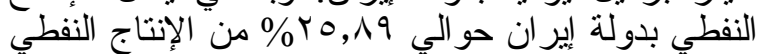
بدول مجلس التعاون الخليجي .وبمقارنة الاحتياطي النفطي الإني

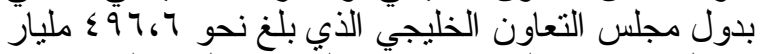

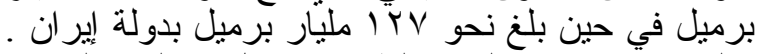

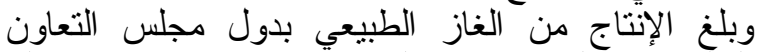

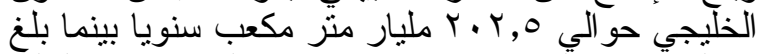

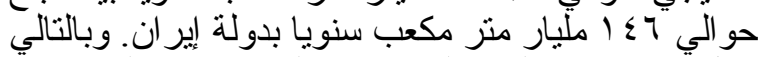

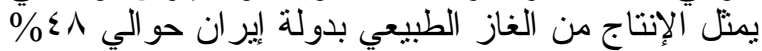

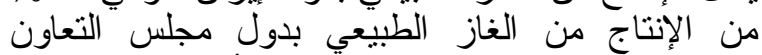

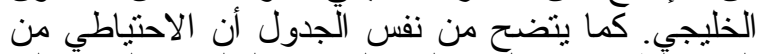

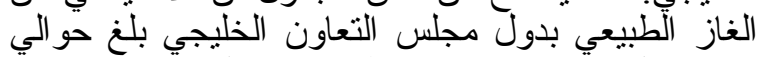

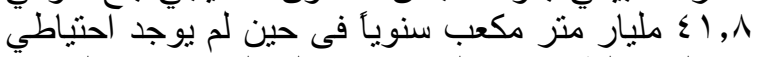

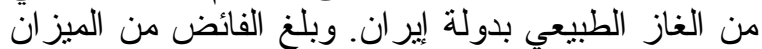

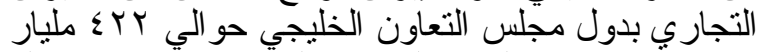

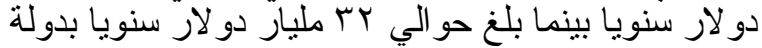

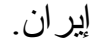

ويتضح من جدول 7 أن قيمة الصادر ات بدول مجلس

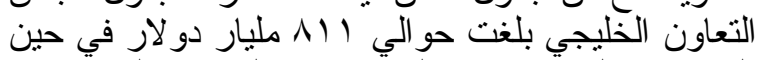

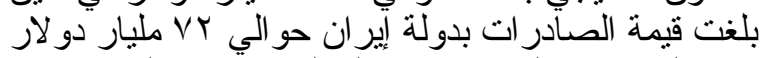

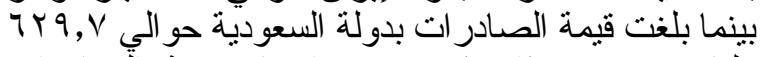

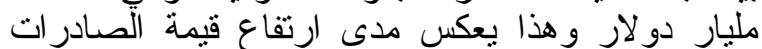

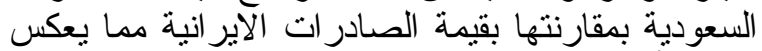

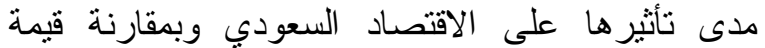

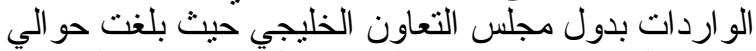

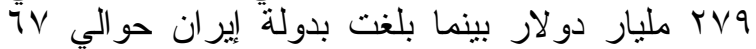

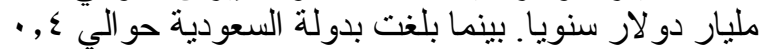

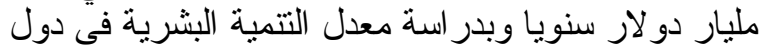

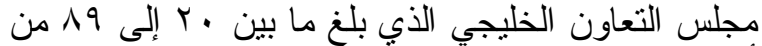

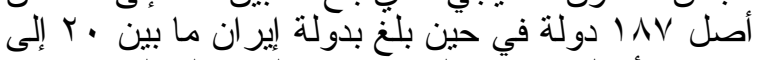

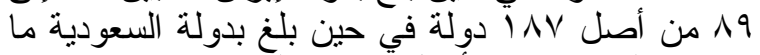

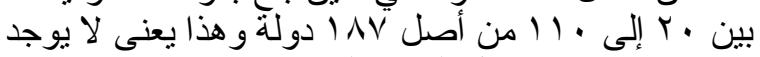
فرق ما بينهم في معدل التتمية البشرية.

إيجاد العلاقة بين محددات التجارة الخارجية على التهي النمو الاقتصادي في إيران توصيف النموذج توصيف متغير ات النموذج

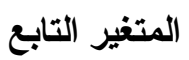

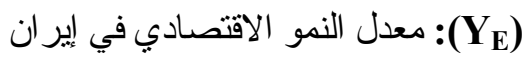
المتفير ات المستقلة: : نسبة مساهمة القيمة المضافة من الصناعات التحويلية من معدل النمو في إير ان. : E2 الإجمالي في إير ان. 
جدول V. يوضح نتأتج العلاقة بين (E)

\begin{tabular}{|c|c|c|c|}
\hline المتغير التابع & 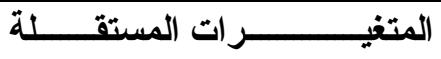 & $\mathbf{R}^{2}$ & الحالة \\
\hline \multirow{5}{*}{$\mathrm{Y}_{\mathrm{E}}$} & $E_{7}, E_{2}, E_{1}, E_{6}, E_{5}, E_{4}, E_{3}$ & 0.629 & 1 \\
\hline & $\mathrm{E}_{7}, \mathrm{E} 2, \mathrm{E}_{1}, \mathrm{E}_{6}, \mathrm{E}_{4}, \mathrm{E}_{3}$ & 0.628 & 2 \\
\hline & $\mathrm{E}_{7}, \mathrm{E}_{1}, \mathrm{E}_{6}, \mathrm{E}_{2}, \mathrm{E}_{3}$ & 0.627 & 3 \\
\hline & $\mathrm{E}_{7}, \mathrm{E}_{1}, \mathrm{E} 6, \mathrm{E}_{2}$ & 0.605 & 4 \\
\hline & $\mathrm{E}_{7}, \mathrm{E}_{6}, \mathrm{E}_{2}$ & 0.570 & 5 \\
\hline
\end{tabular}

المصدر: جمعت وحسبت من جدول رقم ؛.

جاءت أR =

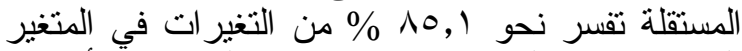

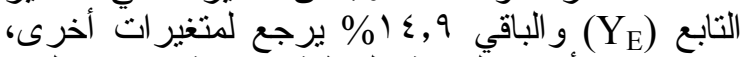

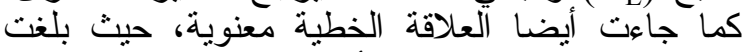
0.000 = Cigf جاءت المتغير ات المستقلة غير معنوية كما هو و اضح

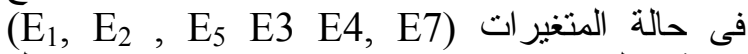

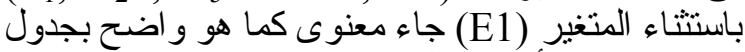
9، وهى نسبة أكبر قليلا من 0

العلاقة بين أهم المتغيرات التي تؤثر على معدل النمو الاقتصادي فى السعودية توصيف النموذج توصيف متغيرات النموذج

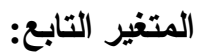

(Y) المتغير ات المستقلة

S S الإجمالي بالسعودية.

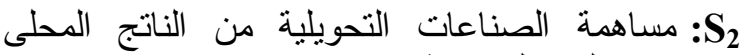
الإجمالي بالسعودية.

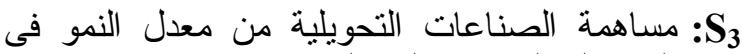
الناتج المحلى الإجمالي بالسعودية.

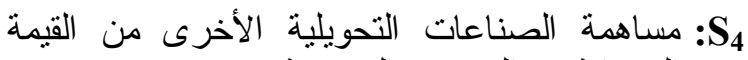
المضافة في التصنيع بالسعودية.

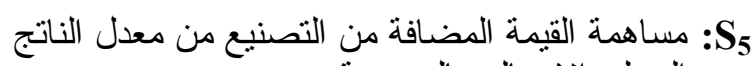
المحلى الإجمالي بالسعودية.

S6 S:

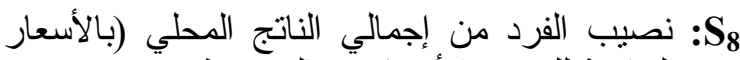
الجارية للاو لار الأمريكي) بالسعودية.
ومن نتائج تقدير العلاقة الخطية بجدول ^ بين معدل

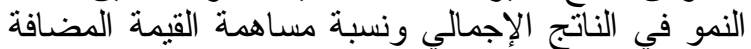

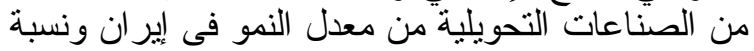

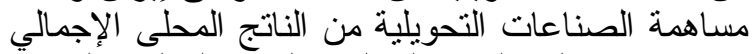

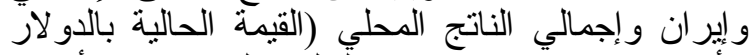

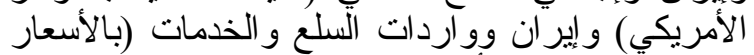

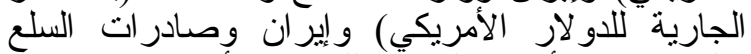
و الخدمات (بالأسعار الجارية للأولارية الار الأمريكي) و إيران

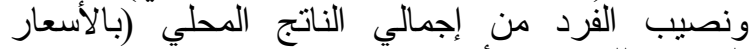

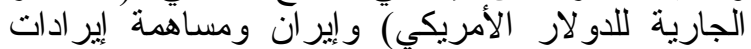

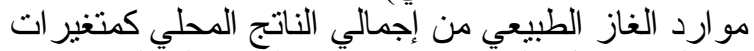

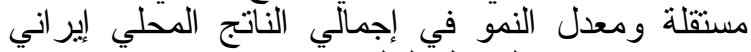
كمتغير تابع، في الجدول الأنالي:

$Y_{E}=a_{0}+a_{1} E_{1}+a_{2} E_{2}+a_{3} E_{3}+a_{4} E_{4}+a_{5}$ E $E_{5}+a_{6} E_{7}$

جاء الثكل الخطى للعلاقة بين ( E (E S $_{7}, E_{6}$ معبر أ عن وجود علاقة طردية بين المتغير ات المستقلة ( $\left.E_{1}, E_{2}, E_{5}, E_{6}\right)$ علاقة عكسية (E) وحيث بلاحظ أن معاملات الانحدار

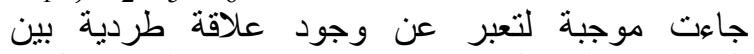
المتغير ات المستقلة (E) (YE) و هو ما يتفق مع افتر اضات النظرية الاقتصادية.

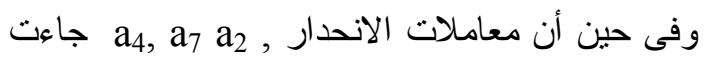
موجبة لتعبر عن وجود علاقينة عكسية بين المتنغيرات

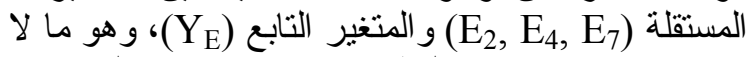

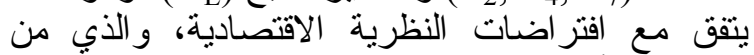

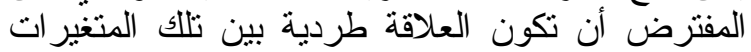

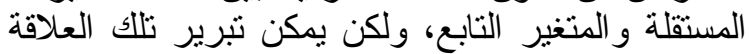

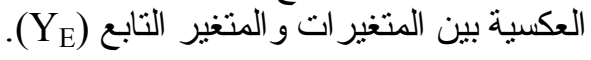
وأخذت العلاقة الصورة التالية: $\mathrm{Y}_{\mathrm{E}}=-5.5+0.84 \mathrm{E}_{1}+0.068 \mathrm{E}_{2}-0.009 \mathrm{E}_{3}-0.004$ $\mathrm{E}_{4}+0.004 \mathrm{E}_{5^{-}}-0.0001 \mathrm{E}_{6}-0.92 \mathrm{E}_{7}$ 
Mahdy and Hassanein | SINAI Journal of Applied Sciences 9 (1) 2020 077-088

جدول ^. يوضح نتائج تقدير العلاقة الخطية بين (1)

\begin{tabular}{|c|c|c|c|c|c|c|}
\hline \multirow[t]{2}{*}{ شكـــلـل العلاقة ــــــة الخطية } & \multicolumn{3}{|c|}{ ثوابت المعادلة ومعنوياتها } & \multicolumn{2}{|c|}{ معنوية العلاقة } & \multirow[t]{2}{*}{$\overline{\mathbf{R}^{2}}$} \\
\hline & & $\mathbf{A}$ & $\mathbf{C i g}_{\mathrm{T}}$ & $\mathbf{F}_{\mathbf{c}}$ & $\mathrm{Cig}_{\mathrm{f}}$ & \\
\hline \multirow{8}{*}{$\begin{array}{c}Y_{E}=a_{0}+a_{1} E_{1}+a_{2} E_{2}+a_{3} E_{3}+a_{4} \\
E_{4}+a_{5} E_{5}+a_{6} E_{6}+a_{7} E_{7}\end{array}$} & $\overline{a_{0}}$ & $-5.512-$ & 0.750 & \multirow{8}{*}{4.2} & \multirow{8}{*}{0.005} & \multirow{8}{*}{0.8510 .152} \\
\hline & $\mathrm{a}_{1}$ & 0.084 & 0.600 & & & \\
\hline & $a_{2}$ & 0.068 & 0.268 & & & \\
\hline & $a_{3}$ & $-9.866 \mathrm{E}-5$ & 0.684 & & & \\
\hline & $\mathrm{a}_{4}$ & $-4.682 \mathrm{E}-7$ & 0.808 & & & \\
\hline & a 5 & $4.288 \mathrm{E}-5$ & 0.822 & & & \\
\hline & $a_{6}$ & 0.0001 & 0.242 & & & \\
\hline & $a_{7}$ & $-0.922-$ & 0.219 & & & \\
\hline
\end{tabular}

جدول 9.

\begin{tabular}{|c|c|c|c|}
\hline المتغير التابع & المتغيــــــــــرات المستقة لــلة & $\mathbf{R}^{2}$ & 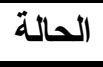 \\
\hline \multirow{7}{*}{$\mathrm{Y}_{\mathrm{S}}$} & $\mathrm{S}_{8}, \mathrm{~S}_{2}, \mathrm{~S}_{5}, \mathrm{~S}_{3}, \mathrm{~S}_{4}, \mathrm{~S}_{6}, \mathrm{~S}_{1}, \mathrm{~S}_{7}$ & 0.596 & 1 \\
\hline & $\mathrm{S}_{8}, \mathrm{~S}_{2}, \mathrm{~S}_{5}, \mathrm{~S}_{3}, \mathrm{~S}_{4}, \mathrm{~S}_{6}, \mathrm{~S}_{1}$ & 0.596 & 2 \\
\hline & $\mathrm{S}_{8}, \mathrm{~S}_{5}, \mathrm{~S}_{2}, \mathrm{~S}_{4}, \mathrm{~S}_{6}, \mathrm{~S}_{1}$ & 0.594 & 3 \\
\hline & $\mathrm{S}_{8}, \mathrm{~S}_{2}, \mathrm{~S}_{4}, \mathrm{~S}_{6}, \mathrm{~S}_{1}$ & 0.591 & 4 \\
\hline & $\mathrm{S}_{8}, \mathrm{~S}_{2}, \mathrm{~S}_{4}, \mathrm{~S}_{1}$ & 0.581 & 5 \\
\hline & $\mathrm{S}_{2}, \mathrm{~S}_{4}, \mathrm{~S}_{1}$ & 0.562 & 6 \\
\hline & $\mathrm{S}_{2}, \mathrm{~S}_{4}$ & 0.554 & 7 \\
\hline
\end{tabular}

وبالتالي يتبين أن أهم تلكاك المتغير ات هي:

S: SW المحلى الإجمالي بالسعودية.

S S2 الإجمالي بالسعودية.

S S الناتج المحلى الإجمالي بالسعودية.

S S المضافة في التصنيع بالسعودية.

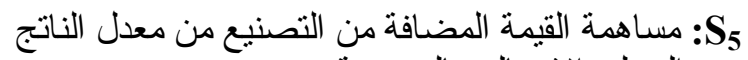
المحلى الإجمالي بالسعودية.

S6: الناتج المحلى الإجمالي بالمليار دو لار بالسعودية. S8 الجارية للاو لار الأمريكي) بالسعودية.

\section{تقدير العلاقة الخطية}

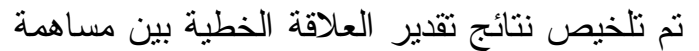

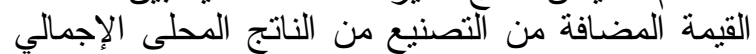
بالسعودية ومساهمة الصناعات التحويلية من الناتجاتئج
الثكل الرياضي للنموذج

يأخذ النموذج الصورة التالية:

$\mathrm{Y}_{\mathrm{S}}=\mathrm{a}_{0}+\mathrm{a}_{1} \mathrm{~S}_{1}+\mathrm{a}_{2} \mathrm{~S}_{2}+\mathrm{a}_{3} \mathrm{~S}_{3}+\mathrm{a}_{4} \mathrm{~S}_{4+} \mathrm{a}_{5} \mathrm{~S}_{5}+\mathrm{a}_{6} \mathrm{~S}_{6}+$ $\mathrm{a}_{7} \mathrm{~S}_{7}+\mathrm{a}_{8} \mathrm{~S}_{8}$

تقدير العلاقة بين أهم المتغيرات التي تؤثر على التئ معدل النمو الاقتصادي في السعودية الميرية

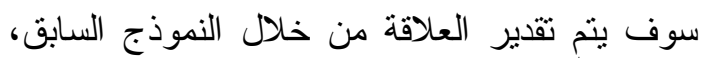

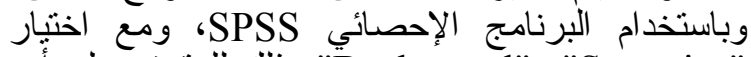

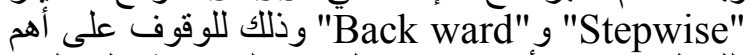

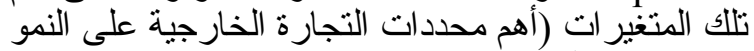

الاقتصادي بالسعودية).

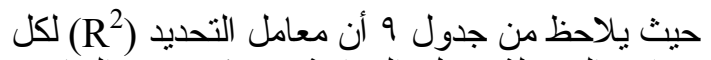

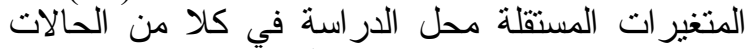

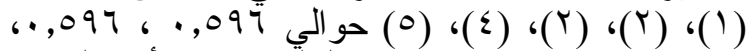

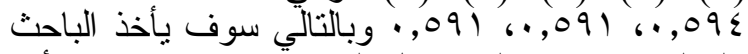

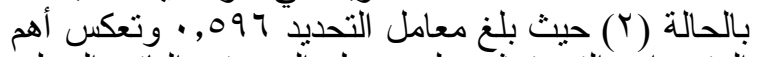

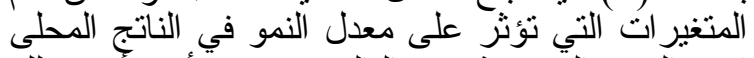

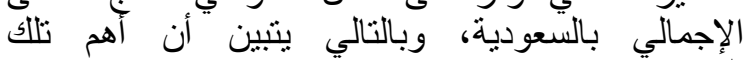

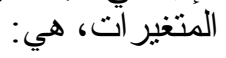


Sت S $\left(\mathrm{Y}_{\mathrm{S}}\right),\left(\mathrm{S}_{6}, \mathrm{~S}_{8}\right.$

$\mathrm{Y}_{\mathrm{S}}=6.6-0.22 \mathrm{~S}_{1}+0.077 \mathrm{~S}_{2}+0.09 \mathrm{~S}_{3}+0.104$ $\mathrm{S}_{4}+0.07 \quad \mathrm{~S}_{5}+0.0005 \mathrm{~S}_{6}+0.001 \mathrm{~S}_{7}$

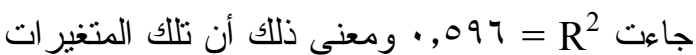
المستقلة تفسر نحو

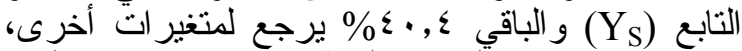
كما جاءت ايضا ألعلاقة الخطية معنوية، حيث بلغير بلغت 0.01 = Cigf

جاءت المتغير ات المستقلة غير معنوية كما هو و اضح

فى حالة المتغير ات هو موضح حجدول • (، و هى نسبة أكبر قليلا من \% \% التوصيات

1 ـ تحديـد مـدى أهميـة السياسـة الخارجيـة الإير انيـة في منطقة الخليج العربي.

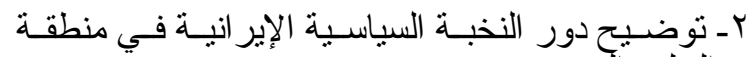
الخليج العربي.

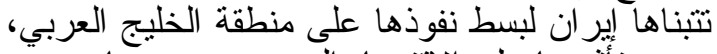

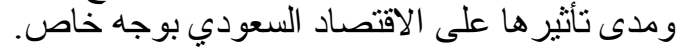

ـ ـ تحديد الإجر اءات السياسية والأمنية و الاقتصادية التي التي

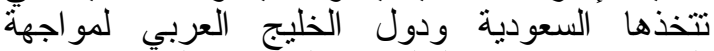
التوجهات الإير انية الخليجية و المشروع النورية

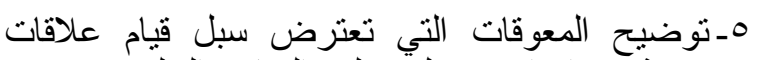
حسنة بين إيران - ودول مجلس التعاون الخليجي. 7- التعاونيح). دور العلاقات الإيرانية الخليجية (دول مجلس ائسل

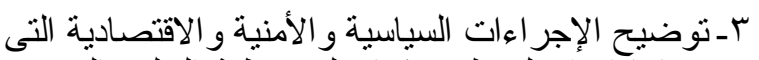

المحلى الإجمالي بالسعودية ومساهمة الصناعات التحويلية

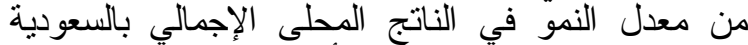

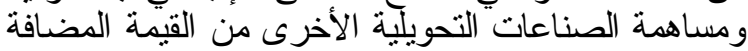

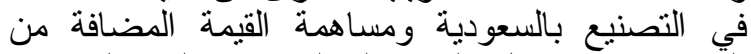
التصنيع من معدل الناتج المحلى الإجمالي بالسية بالسعودية لإلية

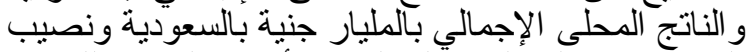

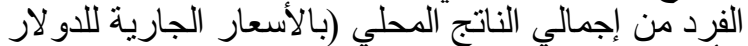

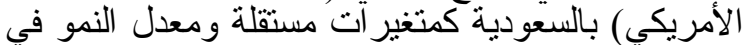

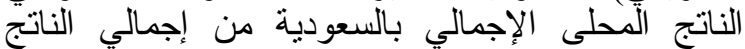

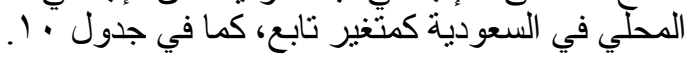
$Y_{S}=a_{0}+a_{1} S_{1}+a_{2} S_{2}+a_{3} S_{3}+a_{4} S_{4}+a_{5} S_{5}+a_{6} S_{8}$

$$
\text { ويلاحظ من خلال الجدول (• (1)، ما يلى: }
$$

جاء الثنكل الخطى للعلاقة بين (

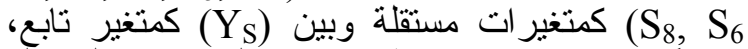
معبر آ عن وجود علاقة طردية بين المتغير ات المستقلّة و المتغير التابع (S ( $\left._{2}, \mathrm{~S}_{2}, \mathrm{~S}_{4}, \mathrm{~S}_{5}, \mathrm{~S}_{6}, \mathrm{~S}_{8}\right)$ وكذللك وجود علاقة عكسية (S) و المتغير التابع (Y) ، وحيث يلاحظ أن معاملات الانحدار a

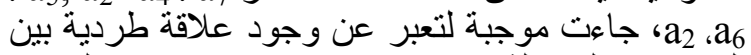
المتغير ات المستقلة

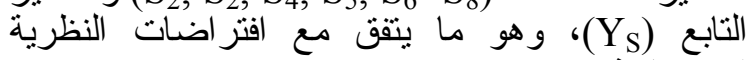

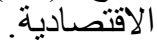

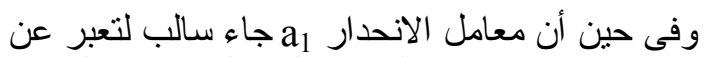

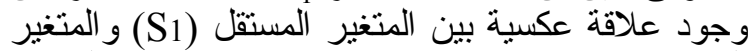

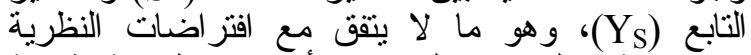

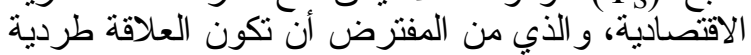

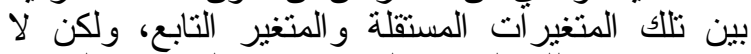

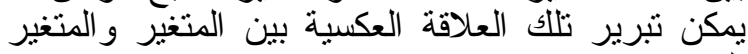

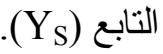
و أخذت العلاقة الصورة التالية: جدول · ا ـ نتائج تقدير العلاقة الخطية

\begin{tabular}{|c|c|c|c|c|c|c|c|}
\hline \multirow{2}{*}{ شكــل العلاقة ـــــــة الخطية } & \multicolumn{3}{|c|}{ ثُوابت المعادلة ومعنوياتها } & \multicolumn{2}{|c|}{ معنوية العلاقة } & \multirow[t]{2}{*}{$\mathbf{R}^{2}$} & \multirow{2}{*}{ dw } \\
\hline & & $\mathbf{A}$ & $\mathrm{Cig}_{\mathrm{t}}$ & $\mathbf{F}_{\mathbf{c}}$ & $\mathbf{C i g}_{\mathrm{f}}$ & & \\
\hline \multirow{8}{*}{$\begin{array}{c}Y_{S}=a_{0}+a_{1} S_{1}+a_{2} S_{2}+a_{3} S_{3}+a_{4} \\
S_{4}+a_{5} S_{5}+a_{6} S_{6}+a_{7} S_{8}\end{array}$} & $\mathrm{a}_{0}$ & 6.571 & 0.552 & \multirow{8}{*}{0.072} & \multirow{8}{*}{0.01} & \multirow{8}{*}{0.596} & \multirow{8}{*}{2.4} \\
\hline & $\mathrm{a}_{1}$ & $-.222-$ & 0.414 & & & & \\
\hline & $a_{2}$ & 0.077 & 0.815 & & & & \\
\hline & $a_{2}$ & 0.090 & 0.442 & & & & \\
\hline & $\mathrm{a}_{4}$ & 0.104 & 0.072 & & & & \\
\hline & $\mathrm{a}_{5}$ & 0.070 & 0.660 & & & & \\
\hline & $a_{6}$ & $5.204 \mathrm{E}-6$ & 0.511 & & & & \\
\hline & $a_{7}$ & 0.001 & 0.275 & & & & \\
\hline
\end{tabular}


وزارة الاقتصاد والتخطيط، (ه بـ إهـ). خطة التتمية

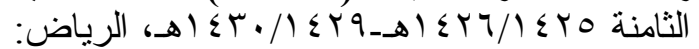

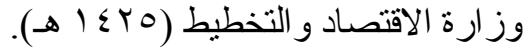

AS-Sudais, A. (1999). The Applicability of the Theory of customs unions: the Case of the Cooperation Council for the Arab States of the Gulf (The GCC).

Calbertc, H. (1994). Middle East, Patterns (Places, people, and politics), Baylor university, west view press, $2^{\text {nd }}$ Ed.

Cherp, A. (2012). Energy and Security, Central European Univ., Budapest، Hungary.

Sajjil (2011). Ballistic Missiles of the World-Iran, missilethread. com, 2011, at: Saleh Al-Khatlan, Saudi-Iranian relations: Implications for Gulf security, in Bridging A Gulf: Peace Building in West Asia, Ed. Majid Tehranian, IB, Tauris, London, 2003.

$$
\begin{aligned}
& \text { إحصائيات منظمة الجمارك الإيرانية (1^ • Y). وكالة } \\
& \text { "مهر " الإير انية. }
\end{aligned}
$$

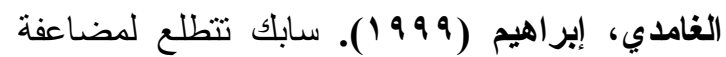

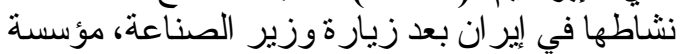
اليمامة الصحفية.

\title{
المُلخص العربي
}

دراسة اقتصادية للتجارة الخارجية الإير انية الخليجية وتأثيرها على الاقتصاد السعودي

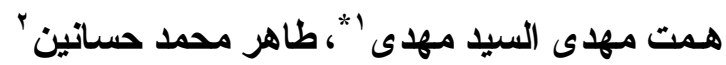

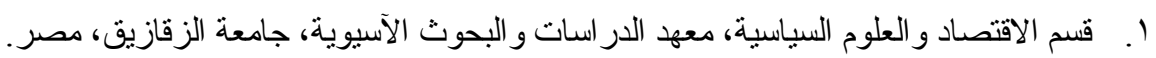

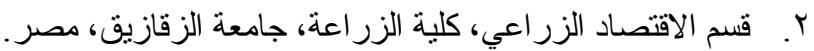

أصبح تحقيق الأمن القومي الخليجي يمنل أهمية كبرى لدول مجلس التعاون الخليجي، لاسيما بعد تتامي التحديات التي

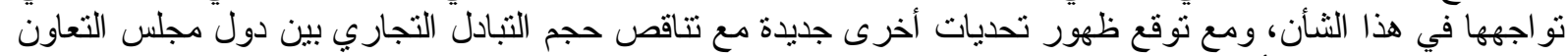

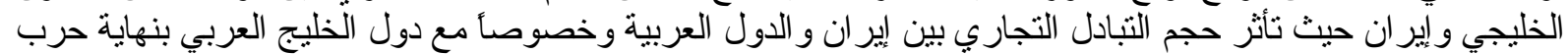

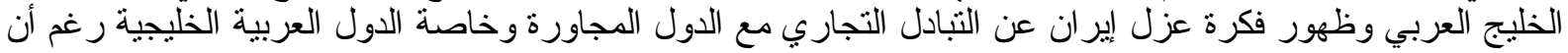

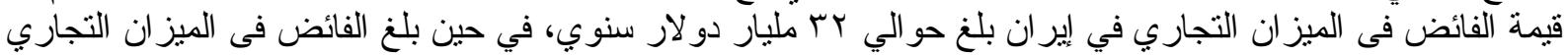

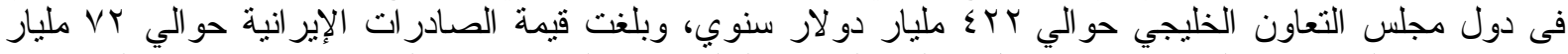

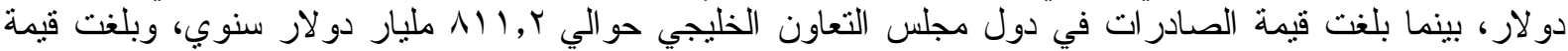

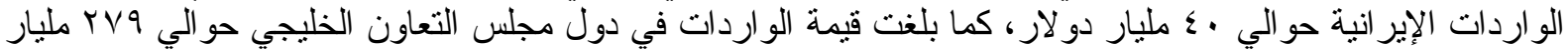

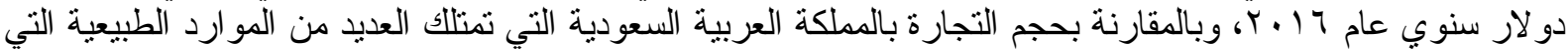

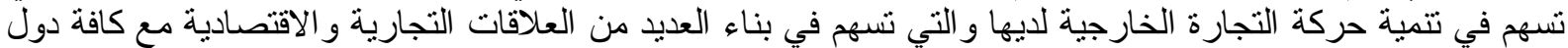

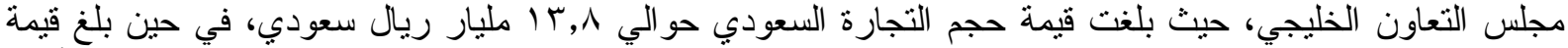

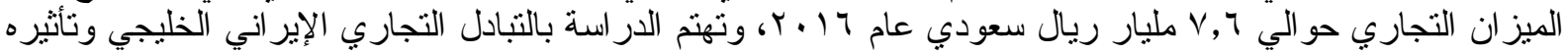

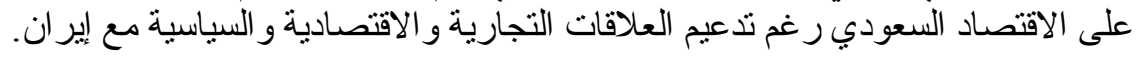

الكلمات الاسترشادية: اقتصاديات التجارة الخارجية، الاقتصاد السعودي، الأمن القومي الخليجي، دول مجلس التس التعاون.

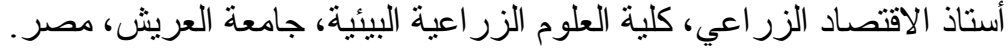
أستاذ الاقتصاد الزر اعي المساعد، كلية العلوم الزر اعية البيئية، جامعة العريش، لئهر، مصر.

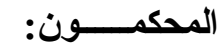

ا ـ أ.د. رياض إسماعيل مصطفي

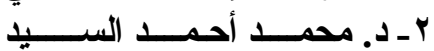

